The alginate wool filter has proved to be very useful for sampling airborne fungi and bacteria which can be cultured. It is particularly suited for work with selective media, since aliquots of the solution of one filter can be plated out on several media. If

desired, counts of non-culturable spores may be obtained by microseopic examination of the sediment obtained on centrifuging an aliquot of the solution of the filter. When the filter is nsed in conjunction with a small hand-operated pump, a simple, efficient and readily portable spore-trap results.

The work was performed at the Asthma and Allergy Research Unit, St. David's Hospital, Cardiff, during the tenure of a research grant from the Asthma Research Council, London. I thank Mr. H. A. Hyde and Dr. D. A. Williams for encouragement, Mr. R. H. McDowell of Alginate Industries, Ltd., for technical advice and Messrs. Courtaulds, Ltd., for supplies of calcium alginate yarn.

National Research Council,

Prairie Regional Laboratory, Saskatoon, Canada. April 19.

3 Hirst, J. M., Ann. App. Biol., 39, 257 (1952).

May, K. R., J. Sci. Instr., 22, 187 (1045).

\section{A Direct Measurement of the Energy Locally Absorbed from a Gamma-Ray Beam}

THE energy locally absorbed in a material exposed to $\gamma$-radiation has usually been determined indirectly from measurements with an ionization chamber, using the Bragg-Gray formula. The calculation involves the use of two quantities the values of which are not accurately known : $W$, the average energy required to produce an ion pair, and $\rho$ the ratio of the stopping powers of the material and the gas. The direct measurement of the energy absorbed presents some difficulties due to the small rise of temperature which is produced. If a dose corresponding to $300 \mathrm{r}$. is given to a sample of water, the temperature will rise by only $6.7 \times 10^{-4} \mathrm{deg}$. C. However, the development of the thermally sensitive resistors known as thermistors has made possible the detection of a change of temperature of less than $10^{-5} \mathrm{deg}$. C.

In this laboratory a radiation calorimeter has been constructed. The sensitive part consists of a cylinder ( $2 \mathrm{~cm}$. in diameter, $2 \mathrm{~cm}$. in length) of a given material in which are imbedded a thermistor and a heating coil. The cylinder is suspended in an evacuated and aluminized glass vessel placed in a tank of water the temperature of which is controlled. To minimize the effects of heat transfer and self-heating in the thermistor, an identical dummy cylinder is suspended in another evacuated vessel but does not receive radiation. The thermistors are connected to the opposite arms of a d.c. Wheatstone bridge. A change in temperature of on $\theta$ of the cylinders produces an unbalanced voltage which is amplified and recorded on a self-balancing potentiometer. For calibration, a known amount of energy is dissipated in the cylinder by the heating coil. The thermistors used have a resistance of the order of $100,000 \mathrm{ohms}$ and a negative temperature coefficient of $-4 \cdot 7$ per cent per deg. at $25^{\circ} \mathrm{C}$. With the present arrangement it is possible to measure a change of temperature of $10^{-8} \mathrm{deg}$. C. with an accuracy better than 1 per cent.

Experiments have been carried out with absorbing cylinders of carbon and aluminium exposed to the beam from our 1,000-curie cobalt-60 unit. The dosage-rate at the position of the cylinders is about 30 r. per minute. After the energy absorption measurements, the cylinders are replaced by air-filled ionization chambers of the same material and same dimensions. The results of a number of experiments are shown in Table 1.

The second column of Table 1 gives the values of the energy absorbed per gm. per min. and is the average of three determinations for each material. The third column shows the corresponding ionization per gm. of air per min. In the fourth column we find the ratio $E_{m} / J_{m}=W p_{m}$. Since air was used as the gas in both chambers, the ratio $\left(\rho_{m}\right) \mathrm{Al} /\left(\mathrm{pm}_{\mathrm{m}}\right) \mathrm{C}$ can be obtained. This mass stopping-power ratio $(0.881)$ can be compared with the values calculated from the theoretical data of Whyte ${ }^{1}(0.886)$ and Spencer ${ }^{2}(0 \cdot 866)$. If we accept Whyte's values for $\rho_{m}$, we obtain the values of $W$ for air shown in the last column. The values are about 2 per cent higher than the usually accepted value of $32 \cdot 5 \mathrm{eV}$./ion-pair and lower than the value of $35 \cdot 0 \mathrm{eV}$./ion pair obtained by Valentine ${ }^{8}$. Further measurements are in progress using other materials (for example, water) and other gases.

H. E. JoHrs

J. P. BERNIER

Department of Physics,

D. V. CoRmack

University of Saskatchewan, and

Saskatoon Cancer Clinic,

Saskatoon, Saskatchowan. April 28.

${ }^{1}$ Whyte, G. N., Nucleonics, 12, No. 2, 18 (1954).

2 Spencer, L. V., and Attix, F. H., Radiology, 64, 113 (1955).

- Valentine, J. M., Proc. Roy. Soc., A, 211, 75 (1952).

\section{Mineralogy and Crystal Structure of Chamosite}

THE occurrence of the hydrous ferrous silicate, chamosite, in sedimentary ironstones was described by Hallimond ${ }^{1}$. Later, with Harvey and Bannister ${ }^{2}$, he concluded that chamosite differed from the ironbearing chlorites. Brindley ${ }^{3,4}$ examined chamosites from laterites and other sources, and concluded that they were of kaolin-type. Many of the variations in X-ray diagrams were accounted for by the existence of two kaolin-type structures, one ortho-hexagonal and one monoclinic, with simply related cell dimensions. In common with some other clay minerals, chamosite had random displacements of layers parallel to the $b$-axis of integral multiples of $b / 3$, giving rise to sharp $(h k l)$ reflexions only for $k=3 n$. Brindley concluded from the presence of $(h k l)$ re:flexions for $h \neq 3 n$ that there were no random $a$-axis 